\title{
Differentially expressed microRNA identification and target gene function analysis in starvation-induced autophagy of AR42J pancreatic acinar cells
}

\author{
BO GAO ${ }^{1}$, DUANPING WANG ${ }^{2}$, WANG SUN ${ }^{1}$, XIANZHI MENG ${ }^{1}$, WEIHUI ZHANG ${ }^{1}$ and DONGBO XUE ${ }^{1}$ \\ ${ }^{1}$ Department of General Surgery, The First Affiliated Hospital of Harbin Medical University, Harbin, Heilongjiang 150001; \\ ${ }^{2}$ Department of General Surgery, The First Hospital of Qiqihar, Qiqihar, Heilongjiang 161005, P.R. China
}

Received May 24, 2015; Accepted March 23, 2016

DOI: $10.3892 / \mathrm{mmr} .2016 .5240$

\begin{abstract}
Acute pancreatitis (AP) is a common acute digestive tract disease, with increased morbidity and mortality, and an unclear pathogenesis. Trypsinogen activation in pancreatic acinar cells may be the primary mechanism underlying the development of AP. Previous studies reported that autophagy participates in the formation of acinar cell vacuoles in AP and in the process of trypsinogen activation as an important cause of AP. Furthermore, microRNAs (miRNAs) maintain the autophagy process by regulating the expression of autophagy-associated genes. In the present study, an in vitro pancreatic acinar cell autophagy model was established using the AR42J starvation-induced pancreatic acinar cell line. Twenty differentially expressed microRNAs were identified using miRNA microarray. Bioinformatics analysis was used to predict the target genes of miRNAs and analyze the functions of differentially expressed miRNAs. The results demonstrated that only the downregulated miRNA rno-miR-148b-3p predicted 593 target genes with a statistical significance $(\mathrm{P}<0.05)$, from which 10 genes were autophagy-associated. The results of gene ontology and pathway analyses demonstrated that the target genes of miRNAs were enriched in the Response to insulin stimulus, Regulation of cell death and the Insulin signaling pathways $(\mathrm{P}<0.05, \mathrm{FDR}<0.05)$. In addition, protein-protein interaction network analysis demonstrated a widespread interaction among the 593 target genes. The results of the present study may provide novel targets for research on the mechanisms of autophagy-promoted AP and AP treatment.
\end{abstract}

Correspondence to: Professor Weihui Zhang or Professor Dongbo Xue, Department of General Surgery, The First Affiliated Hospital of Harbin Medical University, 23 You Zheng Street, Harbin, Heilongjiang 150001, P.R. China

E-mail: zhangweihui626@hotmail.com

E-mail: xue9971@sina.com

Key words: acute pancreatitis, autophagy, microRNA, bioinformatics, AR42J

\section{Introduction}

Acute pancreatitis (AP) is a common acute digestive tract disease, associated with high rates of morbidity and mortality. The pathogenesis of AP remains unclear; however, trypsinogen activation is considered to be an important cause and early event in the development of AP. Its activation in pancreatic acinar cells has been suggested to be the primary mechanism underlying the development of AP (1). A previous study suggested that advanced activation of intracellular trypsinogen may be associated with an abnormal calcium signaling pathway, low extracellular $\mathrm{pH}$, lysosome activation and plasminogen aggregation (2). A previous study demonstrated that autophagy and changes in lysosomal cathepsin are associated with the occurrence of AP (3), which has resulted in this area becoming a focus of research.

Autophagy, a form of programmed cell death, involves the formation of autophagosomes by the engulfing of cellular degradation products with single- or double-layered membranes. The products are then transported to lysosomes to form autolysosomes, and are digested and degraded by intralysosomal hydrolases to complete the cell metabolism and renewal of cell organelles (4). A number of studies have shown that autophagy is associated with the pathogenesis of AP. Sherwood et al (5) demonstrated that during early AP, trypsinogen activation occurred in pancreatic acinar vacuoles, suggesting that autophagy may be involved in the pathogenesis of AP. During autophagy, trypsinogen is transported to the lysosomes, which have an acidic environment, resulting in zymogen activation (5). Mareninova et al (6) detected abnormal autophagy in an in vitro AP rat model, in which an increased number of zymogens accumulated in autolysosomes, resulting in trypsinogen activation. These studies suggested that autophagy participates in the formation of acinar cell vacuoles in AP and in the process of trypsinogen activation, therefore, autophagy may be an important cause of AP.

MicroRNAs are non-coding endogenous RNAs expressed in eukaryotes and are typically 18-25 nucleotides in length. Mature microRNAs bind to the 3'-untranslated region (UTR) of target genes through complementary base pairing to degrade the target mRNA or inhibit the translation of the mRNAs to regulate gene expression. Thus, miRNAs post-transcriptionally 
regulate gene expression (7). It has been demonstrated that miRNAs have regulatory functions in numerous pathological and physiological processes, including cell proliferation, differentiation and cell death. Recent studies have confirmed that miRNAs maintain the autophagy process by regulating the expression of autophagy-related genes, which may result in autophagy inhibition $(8,9)$. By contrast, selected miRNAs activate autophagy $(10,11)$. Tekirdag et al (12) demonstrated that miR-181a may downregulate the mRNA level and protein expression of the autophagy-related gene ATG5 through binding to the 3'-UTR of ATG5. miR-181a thus blocks the formation of the ATG12-ATG5-ATG16L1 complex and inhibits the occurrence of autophagy (12). Korkmaz et al (13) demonstrated that autophagy induced by starvation may be inhibited upon transfection of miR-376b into the MCF-7 breast cancer cell line and the Huh-7 human liver cancer cell line. In addition, miR-101, the miR-30 family and miR-130a were demonstrated to be associated with autophagy (14-16). However, it is unknown which miRNAs are capable of regulating autophagy in the pancreatic acinar cells. As autophagy is associated with trypsinogen activation in the pancreatic acinar cells in AP and a variety of miRNAs may regulate autophagy, identifying differential expression of miRNAs and their functions in pancreatic acinar cell autophagy may be important in the understanding of AP pathogenesis and in the development of novel treatments.

The present study established an in vitro pancreatic acinar cell autophagy model using the AR42J starvation-induced pancreatic acinar cell line. The differential expression of miRNAs in AR42J cells during autophagy was detected using miRNA chips. Bioinformatics methods were used to analyze the functions of differentially expressed miRNAs with the aim of identifying novel targets for AP treatment and a greater understanding of autophagy-promoted AP.

\section{Materials and methods}

Cell lines and cell culture conditions. The AR42J rat pancreatic acinar cell line (China Center for Type Culture Collection, Wuhan, China) was cultured in Ham's F12K medium (Sigma-Aldrich, St. Louis, MO, USA) supplemented with $10 \%$ Gibco fetal bovine serum and Gibco antibiotics (100 U/ml of penicillin and $100 \mathrm{mg} / \mathrm{ml}$ of streptomycin) from Thermo Fisher Scientific, Inc. (Waltham, MA, USA), and maintained in a $5 \% \mathrm{CO}_{2}$ and $37^{\circ} \mathrm{C}$ incubator.

Establishment of autophagy model in AR42J cells. In the current study, AR42J cells were treated with Earle's Balanced Salt Solution (EBSS; Leagene Biotech Co., Ltd., Beijing, China) to establish an in vitro autophagy model of pancreatic acinar cells. EBSS is a balanced salt solution that maintains cell activity for a short time in a $\mathrm{CO}_{2}$ environment. EBSS maintains the osmotic pressure and activity of cells, without providing nutrients. Therefore, EBSS is widely used in the preparation of cell starvation models. For the autophagy group in the present study, AR42J pancreatic acinar cells growing at the logarithmic phase were collected. The original medium was discarded, the cells were rinsed gently three times with phosphate-buffered saline solution, and EBSS ( $5 \mathrm{ml} / 25 \mathrm{~cm}^{2}$ cell culture flask) was added to the AR42J cells for 2,4 and $6 \mathrm{~h}$ at $37^{\circ} \mathrm{C}$ with an atmosphere of 5\% humidified $\mathrm{CO}_{2}$. The cells were then harvested using Gibco trypsin (Thermo Fisher Scientific, Inc.), and RNA and protein were extracted using TRIzol and protein lysis buffer, respectively (Invitrogen; Thermo Fisher Scientific, Inc.). For the control group, AR42J cells growing at the logarithmic phase were collected, and Gibco complete nutrient medium (Thermo Fisher Scientific, Inc.) was added for 2, 4 and $6 \mathrm{~h}$.

Western blot analysis. AR42J cells were homogenized in the protein lysis buffer, and the debris was removed by centrifugation at $12,000 \mathrm{x} \mathrm{g}$ for $10 \mathrm{~min}$ at $4^{\circ} \mathrm{C}$. The bicinchoninic acid assay (Thermo Fisher Scientific, Inc.) was used to detect protein concentration according to the protocol of Walker (17). Proteins $(50 \mu \mathrm{g} /$ lane) were separated by $12 \%$ sodium dodecyl sulfate-polyacrylamide gel electrophoresis and transferred onto Invitrogen polyvinylidene fluoride membranes (Thermo Fisher Scientific, Inc.). The membranes were then washed with Tris-buffered saline (10 mM Tris and $150 \mathrm{mM} \mathrm{NaCl}$ ) containing $0.05 \%$ Tween-20 (TBST; Sigma-Aldrich) and blocked with $3 \%$ bovine serum albumin (BSA; Sigma-Aldrich) for $1 \mathrm{~h}$ on an orbital shaker at room temperature. Rabbit polyclonal beclin-1 (1:1,000; sc-11427), LC3-II (1:1,000; sc-28266) and GAPDH $(1: 1,000 ;$ sc-25778) antibodies were purchased from Santa Cruz Biotechnology, Inc. (Dallas, TX, USA). Goat anti-rabbit IgG horseradish peroxidase-conjugated secondary antibodies (1:2,000; ZDR-5306) were purchased from ZSGB Biotechnology (Beijing, China). TBST with 3\% BSA was used to dilute the antibodies. Membranes were incubated with primary antibodies overnight at $4^{\circ} \mathrm{C}$ then washed in TBST for 30 min 3 times. Following washes, membranes were incubated with the secondary antibodies for $2 \mathrm{~h}$ at room temperature, and washed again in TBST for 30 min 3 times. Proteins were visualized by enhanced chemiluminescence (ECL), with an Amersham ECL Plus kit (GE Healthcare Life Sciences, Chalfont, UK). After mixing $1 \mathrm{ml}$ ECL reagents $\mathrm{A}$ and $\mathrm{B}$, the membrane was incubated with the mixing ECL for $2 \mathrm{~min}$, according to the manufacturer's instructions. GAPDH protein levels were used as a loading control. X-ray film (Thermo Fisher Scientific, Inc.) and a Medical Film Processor (AFP Imaging Corporation, Mount Kisco, NY, USA) were used to develop protein bands in the dark. After using an Epson Perfection 3490 Photo scanner (Epson America, Inc, Plainfield, IN, USA) to obtain the TIF images of the protein bands, Image $\mathbf{J}$ software, version 1.49 (imagej.nih.gov/ij/) was used to quantify the expression. The time point of maximum autophagy was investigated for the following miRNA experiments.

RNA extraction. Total RNA of the AR42J cells treated with EBSS (6 h) was extracted and isolated using TRIzol reagent according to the manufacturer's instructions. RNA quantity and quality were measured using the NanoDrop ND-1000 spectrophotometer (Thermo Fisher Scientific, Inc.) according to the manufacturer's instructions with the following parameters: OD A260/A280 ratio, 2.0 for pure RNA (ratios between 1.8 and 2.1 were acceptable); and OD A260/A230 ratio, $>1.8$. RNA integrity was assessed by standard denaturing agarose gel electrophoresis.

miRNA microarray. Following RNA extraction, the miRCURY LNA microRNA, Hi-Power Labeling kit, 
Hy3/Hy5 (Exiqon A/S, Vedbaek, Denmark) was used for miRNA labeling according to the manufacturer's instructions. Briefly, $1 \mu \mathrm{g}$ of each sample was 3'-end-labeled with the Hy3TM fluorescent label, using the T4 RNA ligase as follows: RNA (diluted in $2.0 \mu \mathrm{l}$ water) was mixed with $1.0 \mu \mathrm{l}$ CIP buffer and CIP (Exiqon A/S). The mixture was incubated for $30 \mathrm{~min}$ at $37^{\circ} \mathrm{C}$, and the interaction of the mixture was terminated by incubation for $5 \mathrm{~min}$ at $95^{\circ} \mathrm{C}$. Following incubation, $3.0 \mu 1$ labeling buffer, $1.5 \mu 1$ fluorescent label (Hy3TM), $2.0 \mu 1$ dimethyl sulfoxide and $2.0 \mu 1$ labeling enzyme were added to the mixture. The labeling reaction was incubated for $1 \mathrm{~h}$ at $16^{\circ} \mathrm{C}$, and terminated by incubation for $15 \mathrm{~min}$ at $65^{\circ} \mathrm{C}$. Upon termination of the labeling procedure, the Hy3TM-labeled samples were hybridized on the miRCURY LNA microRNA Array system (version 18.0; Exiqon A/S) according to the manufacturer's instructions. The total $25 \mu 1$ mixture from the Hy3TM-labeled samples with $25 \mu$ l hybridization buffer were first denatured for $2 \mathrm{~min}$ at $95^{\circ} \mathrm{C}$, incubated on ice for $2 \mathrm{~min}$ and then hybridized on the miRCURY LNA microRNA Array system for 16-20 h at $56^{\circ} \mathrm{C}$ in a NimbleGen 12-Bay Hybridization system (Roche Diagnostics, Basel, Switzerland). Following hybridization, the slides were created, washed numerous times with the wash buffer (Exiqon A/S) and dried using centrifugation for $5 \mathrm{~min}$ at $35.7 \mathrm{x} \mathrm{g}$. The slides were then scanned using the Axon GenePix 4000B microarray scanner (Molecular Devices, LLC, Sunnyvale, CA, USA). Scanned images were imported into the GenePix Pro 6.0 software (Molecular Devices, LLC) for grid alignment and data extraction. Replicated miRNAs were averaged and miRNAs with intensities $\geq 30$ in all samples were selected for calculation of normalization factor. Expressed data were normalized using the median normalization. Following normalization, differentially expressed miRNAs were identified through P-value filtering in the R package siggenes (18). The Treeview software, version 1.6 (GubuSoft, LLC, Belmont, MA, USA) was used to conduct the cluster analysis of differentially expressed miRNAs.

Reverse transcription-quantitative polymerase chain reaction $(R T-q P C R)$. miRNA expression levels were detected in the autophagy and control groups by RT-qPCR. Samples were selected from the original microarray experiments for further RT-qPCR testing based on sufficient RNA remaining. cDNA synthesis for RT-qPCR quantification of miRNAs was performed using the TaqMan MicroRNA Reverse Transcription kit (Invitrogen; Thermo Fisher Scientific, Inc.), according to the protocol of the manufacturer. RT was performed using $3 \mu \mathrm{l}$ custom-designed miRNA-specific primers (Invitrogen; Thermo Fisher Scientific, Inc.). An RT master mix was prepared as follows: $3 \mu 1$ RT primer, 5 ng total RNA and 50 units of MultiScribe Reverse Transcriptase (Invitrogen; Thermo Fisher Scientific, Inc.), $1.5 \mu 1$ RT Buffer (10X), $0.15 \mu 1$ dNTPs (100 mM) and 4 units of RNase inhibitor (all from TaqMan kit) were incubated in PCR a tube for $16^{\circ} \mathrm{C}$ for $30 \mathrm{~min}, 42^{\circ} \mathrm{C}$ for $30 \mathrm{~min}$ and $85^{\circ} \mathrm{C}$ for $5 \mathrm{~min}$. The cDNA product was then stored at $4^{\circ} \mathrm{C}$. The sequencing information of selected miRNAs was obtained using a TaqMan miRNA assay (Invitrogen; Thermo Fisher Scientific, Inc.).
The primers were designed as follows: rno-miR-148b-3p, 5'-ucagugcaucacagaacuuugu-3'; rno-miR-6215, 5'-uuuaggguu gcagagccagg-3'; rno-miR-200a-3p, 5'-uaacacugucugguaacg augu-3' and rno-miR-425-5p, 5'-aaugacacgaucacucccguuga-3'. The RT-qPCR was performed using the LightCycler system (Roche Diagnostics). The $20 \mu 1$ reaction mixture contained $1.0 \mu 1$ TaqMan Small RNA Assay (20X), $1.33 \mu 1$ product from the RT reaction, $10.0 \mu \mathrm{l}$ TaqMan Universal PCR Master mix II (2X) and $7.67 \mu 1$ nuclease-free water. The reactions were incubated in 96-well optical plates. PCR conditions were as follows: $10 \mathrm{~min}$ hold at $95^{\circ} \mathrm{C}$, followed by 40 cycles of $95^{\circ} \mathrm{C}$ for $15 \mathrm{sec}$ and $60^{\circ} \mathrm{C}$ for $60 \mathrm{sec}$. GAPDH expression was used as an internal control and the relative expression of each miRNA was determined by the $\Delta \Delta \mathrm{Cq}$ method, comparing expression of the test miRNA to average GAPDH expression, followed by comparison with the autophagy group to the control group (19).

Bioinformatics analysis. Two databases, miRecords (c1.accurascience.com/miRecords) and miRTarBase (mirtarbase.mbc.nctu.edu.tw/) were utilized to screen the known significant target genes of miRNAs. The miRecords is a database of animal miRNA-mRNA interactions, including the manual collection of experimental verification of interactions. Thus far, the miRecords database has collected 548 miRNAs from 9 species and their verified target genes (20). The miRTarBase database comprehensively collects experimental verification of interactions. The latest data demonstrated that the database has collected 773 microRNAs and their 2,632 target genes from 14 species (21). Only differentially expressed genes and miRNAs may be screened as miRNA-mRNA interactions (P-value cut-off was 0.01). Following integration of the data from the two databases, Cytoscape 2.8.2 software (www.cytoscape.org) was used to generate the miRNA-mRNA network. The Human Protein Reference Database (www.hprd.org/) was used to obtain a protein-protein interaction (PPI) network that included 36,874 edges and 9,453 nodes. The target genes were mapped to the PPI network, and pairs of interacting genes in which both genes were differentially expressed were noted, creating sub-networks using the String software, version 10.0 (www.string-db.org). The Gene Ontology (GO) categories (geneontology.org) comprise three structured networks of defined terms to describe gene product attributes (P-value cut-off was 0.05). Based on the latest data from the Kyoto Encyclopedia of Genes and Genomes (KEGG) database (www.genome.jp/kegg), a pathway analysis was conducted using the differentially expressed genes (P-value and FDR cut-offs were 0.05). Literature mining was conducted to determine which of the target genes were autophagy-associated. Briefly, the Perl language was utilized to write a literature review program using the ActivePerl software, version 5.16.2 (ActiveState Software, Inc., Vancouver, BC, Canada). The software was then used to retrieve literature data from the PubMed database (www.ncbi.nlm.nih.gov/pubmed). The search locations were the titles and abstracts of publications, and the search keywords were the names of the differentially expressed genes, and the word 'autophagy'. Finally, manual screening was used to screen for autophagy-associated genes accurately. 

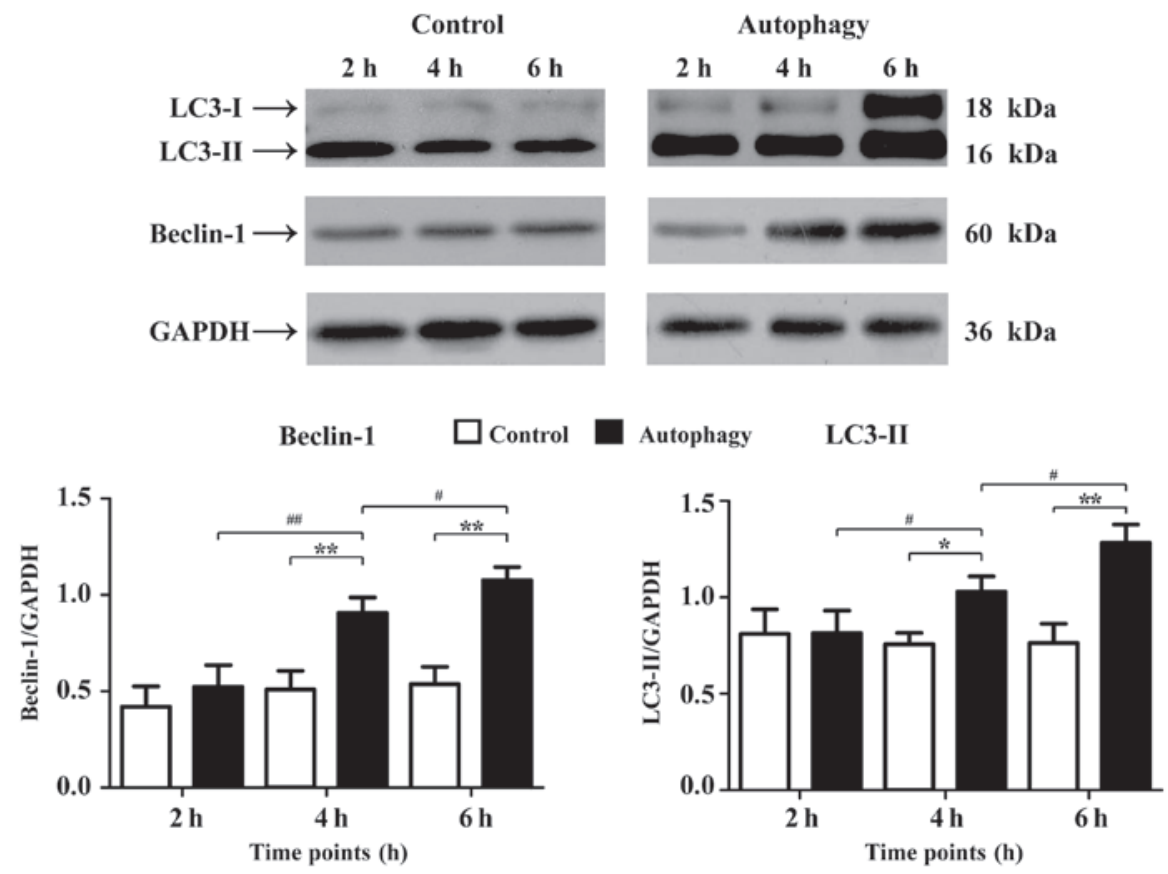

Figure 1. Detection of autophagy in AR42J cells. Western blot analysis was used to detect the protein expression levels of LC3-II and Beclin-1. Autophagy group: AR42J cells treated with EBSS balanced salt solution for 2, 4 and $6 \mathrm{~h}$; control group: AR42J cells treated with complete nutrient medium for 2, 4 and 6 h. ${ }^{\# P}<0.05,{ }^{\# \#} \mathrm{P}<0.01,{ }^{*} \mathrm{P}<0.05$ and ${ }^{* *} \mathrm{P}<0.01$. LC3, microtubule-associated protein 1 light chain 3; GAPDH, glyceraldehyde 3-phosphate dehydrogenase.

Statistical analysis. Data are expressed as the mean \pm standard deviation and comparisons were made using Student's t-test. $\mathrm{P}<0.01$ was considered to indicate a statistically significant difference in gene expression according to the microarray manufacturer's analysis (Exiqon A/S). Categorical variables were given as numbers and percentages, and Fisher's exact test was utilized to test their associations. Statistical analyses of western blotting and RT-qPCR data were performed using the SPSS 17.0 software (SPSS, Inc., Chicago, IL, USA), and $\mathrm{P}<0.05$ was considered to indicate a statistically significant difference.

\section{Results}

Detection of autophagy in the AR42J cells. The results of the western blot analysis (Fig. 1) demonstrated that the expression intensity values of microtubule-associated protein 1 light chain 3 (LC3-II) were $0.81 \pm 0.13$ and $0.82 \pm 0.12$ ( 2 h); $0.76 \pm 0.06$ and $1.03 \pm 0.08(4 \mathrm{~h})$; and $0.76 \pm 0.10$ and $1.28 \pm 0.10(6 \mathrm{~h})$ for the control and autophagy groups, respectively. At the 4 and $6 \mathrm{~h}$ points, the expression of LC3-II in autophagy group was significantly increased compared with that in control group ( $4 \mathrm{~h}, \mathrm{P}<0.05 ; 6 \mathrm{~h}, \mathrm{P}<0.01$ ). The expression of LC3-II gradually increased in the autophagy groups in a time-dependent manner $(\mathrm{P}<0.05)$. Furthermore, the expression intensity values of Beclin-1 were $0.42 \pm 0.11$ and $0.52 \pm 0.11(2 \mathrm{~h}) ; 0.51 \pm 0.10$ and $0.91 \pm 0.08(4 \mathrm{~h})$; and $1.08 \pm 0.07(6 \mathrm{~h})$, for the control and autophagy groups, respectively. At the 4 and $6 \mathrm{~h}$ points, the expression level of beclin-1 in the autophagy group was significantly increased compared with that in control group $(\mathrm{P}<0.01)$. The expression of LC3-II gradually increased in the autophagy groups in a time-dependent manner. The expression of beclin-1 at $4 \mathrm{~h}$ was significantly increased compared with that at $2 \mathrm{~h}(\mathrm{P}<0.01)$ and at $6 \mathrm{~h}$ was higher than that at $4 \mathrm{~h}$ $(\mathrm{P}<0.05)$.

Differentially expressedmiRNAs in AR42J autophagy-induced cells. The results of miRNA microarray analysis demonstrated that 20 miRNAs were differentially expressed (13 upregulated; 7 downregulated). The results of cluster analysis showed that there was expression correlation among these differentially expressed miRNAs (Fig. 2A).

Validation of the expression level of miRNA in AR42J autophagy-induced cells. The result of RT-qRCR detection of the selected miRNAs showed that the expression levels of two miRNAs, rno-miR-6215 (1.88 \pm 0.45$)$ and rno-miR-200a-3p $(2.76 \pm 0.50)$, were significantly upregulated. In addition, the expression levels of rno-miR-148b-3p (0.54 \pm 0.24$)$ and rno-miR-425-5p (0.42 \pm 0.17$)$ were significantly downregulated in the AR42J autophagy-induced cells (Fig. 2B) compared with control. The result was consistent with the results of the microarray. According to the principle of targeted gene suppression by miRNAs, the inhibitory effect of the downregulated miRNA on the target gene was reduced, resulting in high expression of the target gene. Therefore, the downregulated miRNAs in autophagy group, such as rno-miR-148b-3p and rno miR $4255 \mathrm{p}$, may increase target gene expression and thus contribute to the progression of autophagy.

Prediction of differentially expressed miRNA target genes and screening of autophagy-associated genes. Following the combination of data from two miRNA databases, miRanda and miRecords, the target genes predicted in the databases as affected genes of differentially expressed miRNAs were selected. As demonstrated in Fig. 3, only the downregulation 
A

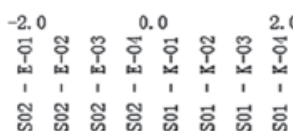

B

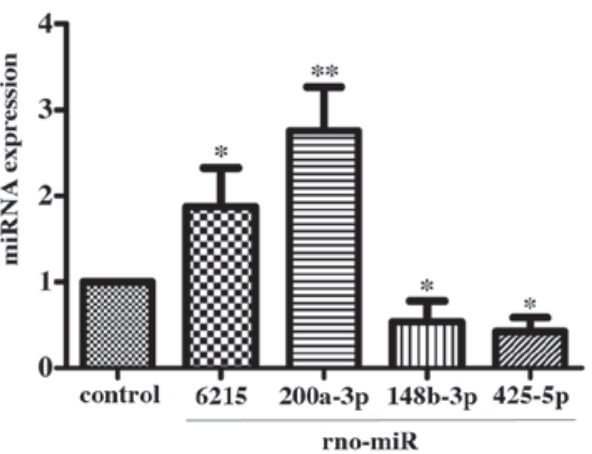

Figure 2. Cluster analysis and validation of differentially expressed miRNAs in the autophagy AR42J-induced cells. (A) Results of cluster analysis (Hotmap) showed that there was an expression correlation among the 20 differentially expressed miRNAs. (B) The expression of four selected miRNAs in the autophagy-induced AR42J cells was detected by reverse transcription-quantitative polymerase chain reaction analysis to validate the results of the miRNA microarray. Rno-miR-6215 and rno-miR-200a-3p were significantly upregulated, and rno-miR-148b-3p and rno-miR-425-5p were significantly downregulated in the AR42J cells. $\mathrm{P}<0.05$ and ${ }^{* *} \mathrm{P}<0.01$ vs. the control group.

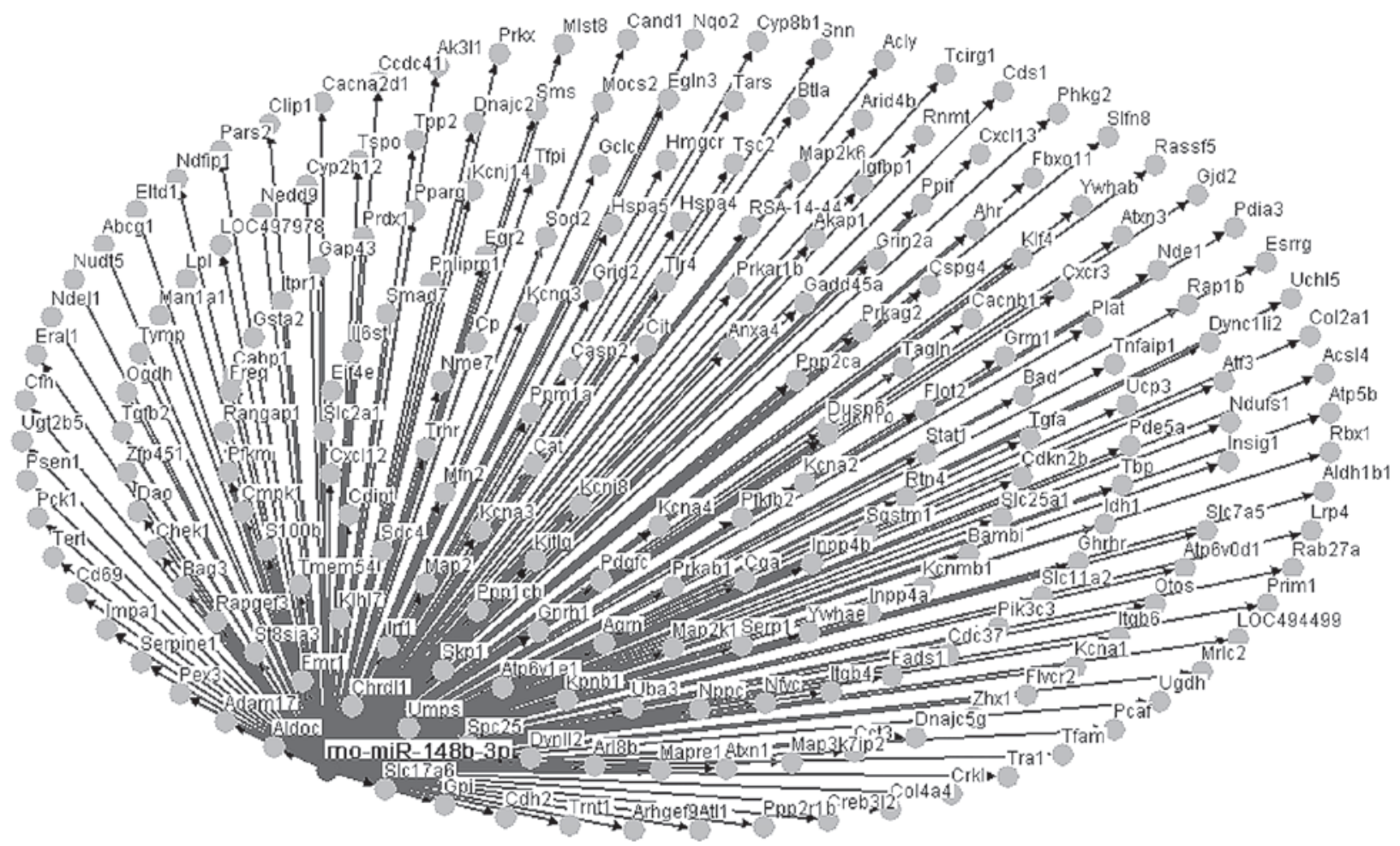

Figure 3. Prediction of differentially expressed miRNA target genes. Two miRNA databases were combined, miRanda and miRecords, and target genes that were predicted in both databases to be genes affected by differentially expressed miRNAs were selected. The results showed that only the downregulated miRNA rno-miR-148b-3p and its 593 target genes were able to satisfy $\mathrm{P}<0.05$.

of miRNA rno-miR-148b-3p and its 593 target genes were shown to be significant $(\mathrm{P}<0.05)$ compared with the control group.

Through literature mining and manual screening, 10 autophagy-related genes from the 593 target genes were identified; Atg12, Hspa5, Hspb6, Sqstm1, Cdc37, Tlr4, Pik3c3, Atf3, Irf1 and Bag3. These genes, directly or indirectly, participated in the autophagy process (Fig. 4).
Results of the target gene $G O$ analysis and pathway enrichment. Using DAVID software (david.ncifcrf.gov), GO function analysis was performed on the target genes. The results demonstrated that the target genes of miRNAs were enriched in the regulation of phosphorylation, regulation of transferase activity, regulation of kinase activity, regulation of protein modification process, response to insulin stimulus, regulation of programmed cell death and regulation of 
Table I. GO analysis.

\begin{tabular}{lllr}
\hline Term & \multicolumn{1}{c}{ Name } & P-value & FDR \\
\hline GO:0042325 & Regulation of phosphorylation & $8.56 \mathrm{E}-10$ & $1.49 \mathrm{E}-06$ \\
GO:0051338 & Regulation of transferase activity & $3.86 \mathrm{E}-08$ & $6.72 \mathrm{E}-05$ \\
GO:0043549 & Regulation of kinase activity & $8.44 \mathrm{E}-08$ & $1.47 \mathrm{E}-04$ \\
GO:0031399 & Regulation of protein modification process & $1.31 \mathrm{E}-06$ & $2.28 \mathrm{E}-03$ \\
GO:0032868 & Response to insulin stimulus & $2.74 \mathrm{E}-06$ & $4.75 \mathrm{E}-03$ \\
GO:0043067 & Regulation of programmed cell death & $1.47 \mathrm{E}-05$ & $2.55 \mathrm{E}-02$ \\
GO:0010941 & Regulation of cell death & $1.59 \mathrm{E}-05$ & $2.77 \mathrm{E}-02$ \\
Pathway analysis & & & \\
rno04910 & Insulin signaling pathway & $7.10 \mathrm{E}-05$ & $8.23 \mathrm{E}-03$ \\
\hline
\end{tabular}

GO, Gene Ontology; FDR, false discovery rate.

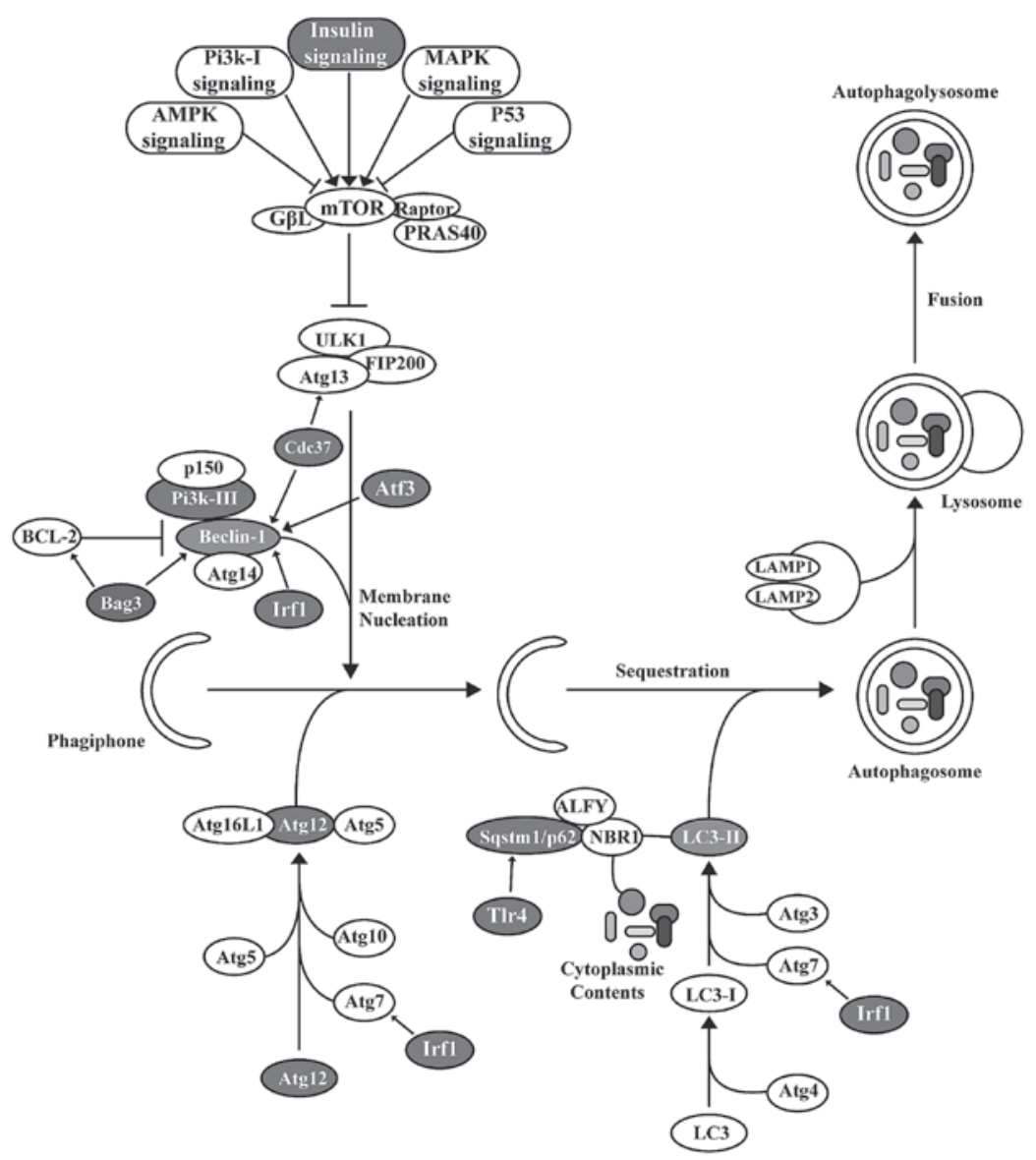

Figure 4. Screening of autophagy-related genes from the target genes and their affect on the autophagy pathway. Through literature mining and manual screening, 10 autophagy-related genes were screened from the 593 target genes: Atg12, Hspa5, Hspb6, Sqstm1, Cdc37, Tlr4, Pik3c3, Atf3, Irf1 and Bag3. All of these genes, directly or indirectly, participated in the autophagy pathway.

cell death pathways. The results analysis of the pathway enrichment of the miRNA target genes using the KEGG database showed that only the insulin signaling pathway simultaneously satisfied the requirements of $\mathrm{P}<0.05$ and FDR $<0.05$ (Table I).

PPI network of target genes. The ultimate goal of the post-genomic era is to recognize the expression patterns and biological functions of all proteins that implement the spectrum of biological activities. One of the key challenges is the analysis of the PPI network, which may increase understanding of cell structures and functions from a systems point of view. At present, the investigation of the PPI network using bioinformatics methods demonstrates significant advantages. In the current study, the String software was utilized to create the PPI network of the 593 target genes (Fig. 5). 


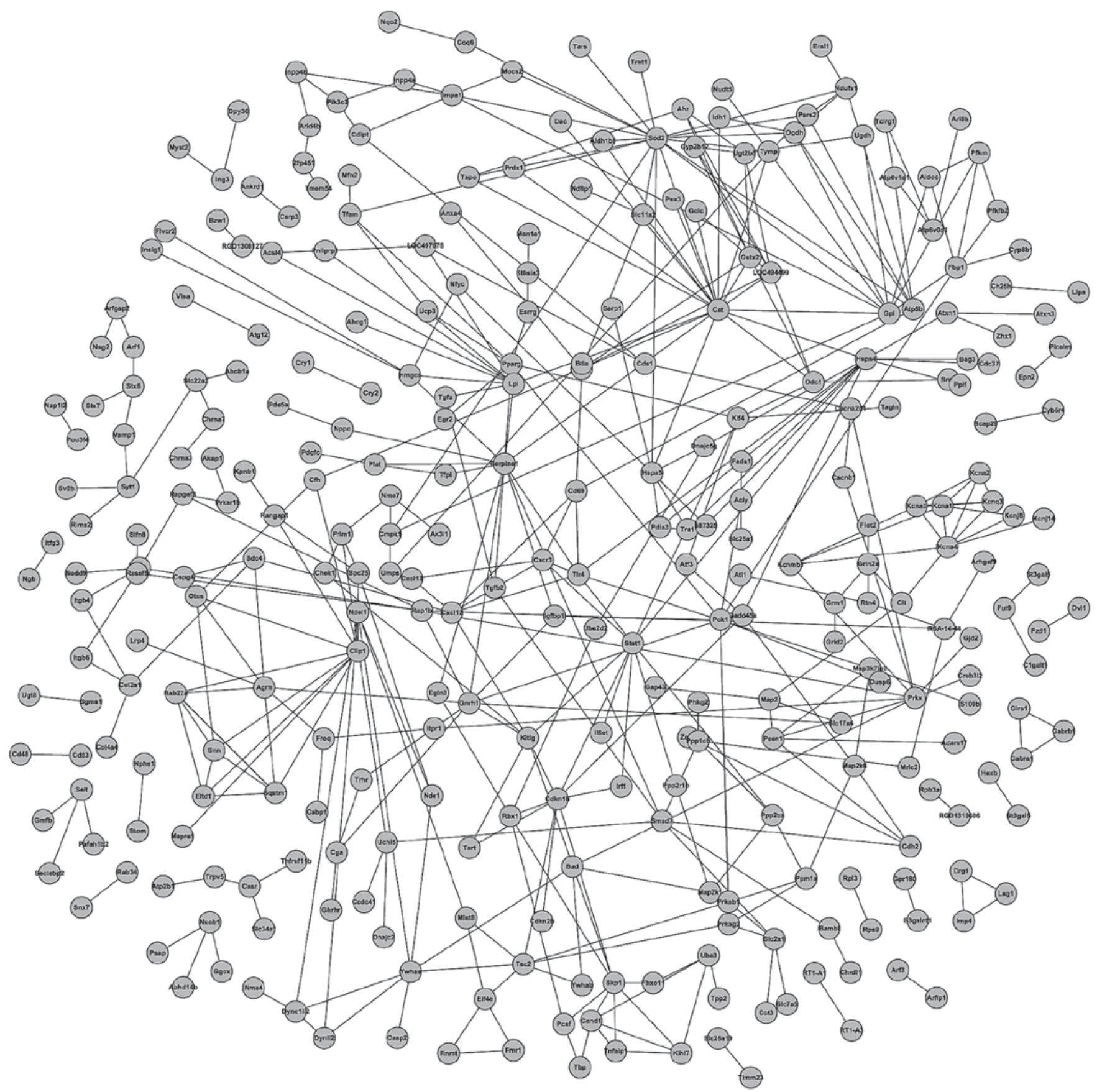

Figure 5. PPI network of target genes. PPI network analysis showed there is a widespread interaction among the 593 target genes of rno-miR-148b-3p. PPI, protein-protein interaction.

\section{Discussion}

Autophagy is a mechanism of self-digestion that represents the only way to degrade organelles on the cellular level. Thus, it has been suggested to be involved in pancreatic self-digestion. Zymogen activation may be the result of lysosomal degradation of zymogen granules during the autophagy process. Hashimoto et al (22) demonstrated that in an ATG5 knockout mouse model of AP, pancreatic acinar cell autophagy was decreased, trypsinogen activity was significantly reduced and the severity of AP was reduced. Thus, it was proposed that the autophagy in the mouse pancreatic acinar cells had a role in damage at the early stages of AP (22). Previous studies demonstrated that a large number of abnormal autophagic vacuoles accumulated within pancreatic acinar cells during AP. A decrease in the lysosomal proteases led to an increase in autolysosomes, and the deficiency and imbalance of lysosomal proteases resulted in a decrease of the degradation capacity of autolysosomes. The accumulation of large amounts of zymogens in autolysosomes resulted in zymogen activation and cell injury (6). These findings support the view of Hashimoto et al (22) that excessive autophagy in pancreatic acinar cells in the early stages of AP exaggerated the degree of the disease. Identification of the changes in gene expression and function of pancreatic acinar cells during autophagy is of great 
significance for studies investigating the mechanisms and treatments of AP.

A variety of proteins are involved in the process of autophagy, including LC3. LC3 is divided into type I and type II proteins. LC3-II is located on the membrane of autophagosomes within cells. The content of this protein is proportional to the number of autophagic vacuoles (23). Beclin-1, another protein in the autophagic process, serves a role in the formation of the pro-autophagic complex and mediates the localization of other autophagy proteins to the autophagosome membrane (24). In the present study, an autophagy model was established using the EBSS starvation method to induce autophagy in the AR42J cells. Western blot analysis demonstrated that the increase in the relative protein expression of LC3-II and Beclin-1 was correlated with the duration of starvation. Starvation-induced autophagy occurred in a time-dependent manner, confirming the successful establishment of an AR42J model of autophagy. It was also observed in order to determine the best time point for subsequent miRNA chip experiments.

The regulation mechanisms of autophagy are complicated. Multiple signaling pathways are known to be involved autophagy, such as the mTOR, insulin and AMPK pathways. miRNAs regulate autophagy through regulating autophagy-related target genes in the above mentioned pathways. It is known that in the process of regulating autophagy, miRNAs mostly inhibit the occurrence of autophagy, while only a few miRNAs are known to activate autophagy. Furthermore, the inhibition of autophagy by miRNAs is mediated by downregulating key molecules of the autophagosome formation process, such as LC3 and Beclin-1, to inhibit the processes of autophagosome formation and membrane extension $(10,11,25)$. The present study identified 20 differentially expressed miRNAs in the AR42J autophagy-induced model and predicted their target genes. Only the downregulated miRNA rno-miR-148b-3p predicted 593 target genes with statistical significance $(\mathrm{P}<0.05)$. Consistent with the fact that miRNAs mediate their effects through the inhibition of their target genes, the expression of rno-miR-148b-3p in the autophagic AR42J cells decreased, leading to a decrease in its ability to inhibit autophagy-associated target genes, thus promoting autophagy. The pathway analysis showed that the target genes of rno-miR-148b-3p were primarily enriched in the insulin signaling pathway. Yamamoto et al (26) demonstrated that the activation of the insulin signaling pathway lead to the removal of accumulated proteins mediated by intracellular macro-autophagy and that this process required the participation of macroautophagy-associated proteins, such as Beclin-1 and hvps34 (26). This result suggests that the low expression of rno-miR-148b-3p during AR42J autophagy may be involved in the induction of autophagy (Fig. 3). The GO analysis results showed that the target genes of rno-miR-148b-3p were primarily involved in the regulation of phosphorylation and other functions, including 'regulation of kinase activity', 'regulation of transferase activity' and 'regulation of cell death'. Autophagy is a programmed cell death pathway, and the process of autophagy depends on the participation of numerous kinases, such as Akt, MAPK and AMPK. These kinases regulate autophagy by phosphorylating downstream autophagy-associated genes $(27,28)$. Therefore, the functions of rno-miR-148b-3p target genes cover a number of processes of autophagy, and the under-expressed rno-miR-148b-3p may be an miRNA regulating the autophagy process.

To further understand the functions of rno-miR-148b-3p in autophagy in the present study, 10 target genes associated with autophagy were screened and the pathways of their participation in the process of autophagy were demonstrated (Fig. 3). Among these target genes, ATG12 and Sqstm1/p62 are most closely associated with autophagy. Previous studies demonstrated that ATG12 and Sqstm1/p62 are necessary factors in the process of autophagy. Atg12, an autophagy-associated gene, is activated by the E1-like enzyme, Atg7, and then transported to the E2-like enzyme, Atg10, to combine with Atg5 and form an autophagosome precursor (29). Sqstm1/p62 was demonstrated to recognize and engulf ubiquitinated proteins flagged for degradation through binding to LC3-II in the process of autophagy (30). Thus, rno-miR-148b-3p may directly participate in the regulation of autophagy through inhibition of Atg12 and Sqstm1/p62. Furthermore, interferon regulatory factor-1 expression was demonstrated to be correlated with that of Atg7 and Beclin-1, and affects the survival of breast cancer cells by regulating autophagy (31). Joo et al (32) demonstrated that $\mathrm{Cdc} 37$ formed a complex with Hsp90 to regulate Atg13-mediated autophagy. Merabova et al (33) indicated that over-expressed Bag3 may increase the interaction between $\mathrm{Bcl} 2$ and Beclin-1 and then induce Beclin-1-dependent autophagy. A previous study demonstrated that TLR4 and p62 mediate lipopolysaccharide-induced autophagy (34). As demonstrated in Fig. 3, the rno-miR-148b-3p target genes indirectly affected autophagy by regulating key genes of the autophagic signaling pathway, further suggesting that rno-miR-148b-3p has the function of regulating autophagy.

Various biological activities are realized by the association and dissociation of protein molecules. The interaction between proteins can form a signal transduction network system that regulates a variety of cellular physiological activities and cellular reactions to the change of microenvironment (35). Therefore, PPI networks can serve an important role in regulating cell activities in different diseases. An in-depth analysis of PPI network would aid the understanding of the progress of disease and its molecular mechanisms (36). The current study demonstrated the interactions among rno-miR-148b-3p target genes through a PPI network analysis. In addition, numerous interactions were indicated among the rno-miR-148b-3p target genes and the autophagy pathway genes. These results suggest that the regulation of autophagy by rno-miR-148b-3p may involve more complicated mechanisms than a single gene or pathway.

In conclusion, the current study established an in vitro model of rat pancreatic acinar cell autophagy in AR42J cells, identified 20 differentially expressed miRNAs using the miRNA chip and demonstrated that rno-miR-148b-3p may be a regulatory miRNA in the autophagic process, using bioinformatics. The results of the present study provide a starting point for studies on the pathogenesis and treatment of AP. However, further research is required to verify the regulatory effects and mechanism of rno-miR-148b-3p and its target genes on autophagy. 


\section{Acknowledgements}

The present study was supported by the National Natural Science Foundation of China (grant nos. 81570579, 81570581, 81370566 and 81170397) and the Harbin Medical University Postgraduate Innovative Research Projects (grant no. YJSCX2015-19HYD).

\section{References}

1. Bhatia M, Wong FL, Cao Y, Lau HY, Huang J, Puneet P and Chevali L: Pathophysiology of acute pancreatitis. Pancreatology 5: 132-144, 2005.

2. Sah RP and Saluja A: Molecular mechanisms of pancreatic injury. Curr Opin Gastroenterol 27: 444-451, 2011.

3. Ohmuraya M and Yamamura K: Autophagy and acute pancreatitis: A novel autophagy theory for trypsinogen activation. Autophagy 4: 1060-1062, 2008.

4. Shimizu S, Yoshida T, Tsujioka M and Arakawa S: Autophagic cell death and cancer. Int J Mol Sci 15: 3145-3153, 2014.

5. Sherwood MW, Prior IA, Voronina SG, Barrow SL, Woodsmith JD, Gerasimenko OV, Petersen $\mathrm{OH}$ and Tepikin AV: Activation of trypsinogen in large endocytic vacuoles of pancreatic acinar cells. Proc Natl Acad Sci USA 104: 5674-5679, 2007.

6. Mareninova OA, Hermann K, French SW, O'Konski MS, Pandol SJ, Webster P, Erickson AH, Katunuma N, Gorelick FS, Gukovsky I and Gukovskaya AS: Impaired autophagic flux mediates acinar cell vacuole formation and trypsinogen activation in rodent models of acute pancreatitis. J Clin Invest 119: 3340-3355, 2009.

7. Ambros V: MicroRNAs and developmental timing. Curr Opin Genet Dev 21: 511-517, 2011.

8. Kim JK, Yuk JM, Kim SY, Jin HS, Yang CS and Jo EK: MicroRNA-125a inhibits autophagy activation and antimicrobial responses during mycobacterial infection. J Immunol 194: $5355-5365,2015$

9. Su M, Wang J, Wang C, Wang X, Dong W, Qiu W, Wang Y, Zhao X, Zou Y, Song L, et al: MicroRNA-221 inhibits autophagy and promotes heart failure by modulating the $\mathrm{p} 27 / \mathrm{CDK} 2 / \mathrm{mTOR}$ axis. Cell Death Differ 22: 986-999, 2015.

10. Jing Z, Han W, Sui X, Xie J and Pan H: Interaction of autophagy with microRNAs and their potential therapeutic implications in human cancers. Cancer Lett 356: 332-338, 2015.

11. Chen Y, Fu LL, Wen X, Liu B, Huang J, Wang JH and Wei YQ: Oncogenic and tumor suppressive roles of microRNAs in apoptosis and autophagy. Apoptosis 19: 1177-1189, 2014.

12. Tekirdag KA, Korkmaz G, Ozturk DG, Agami R and Gozuacik D MIR181A regulates starvation- and rapamycin-induced autophagy through targeting of ATG5. Autophagy 9: 374-385, 2013.

13. Korkmaz G, le Sage C, Tekirdag KA, Agami R and Gozuacik D: miR-376b controls starvation and mTOR inhibition-related autophagy by targeting ATG4C and BECN1. Autophagy 8: 165-176, 2012.

14. Frankel LB, Wen J, Lees M, Høyer-Hansen M, Farkas T, Krogh A, Jäättelä M and Lund AH: microRNA-101 is a potent inhibitor of autophagy. EMBO J 30: 4628-4641, 2011.

15. Kovaleva V, Mora R, Park YJ, Plass C, Chiramel AI, Bartenschlager R, Döhner H, Stilgenbauer S, Pscherer A, Lichter P and Seiffert M: miRNA-130a targets ATG2B and DICER1 to inhibit autophagy and trigger killing of chronic lymphocytic leukemia cells. Cancer Res 72: 1763-1772, 2012

16. Yang X, Zhong X, Tanyi JL, Shen J, Xu C, Gao P, Zheng TM, DeMichele A and Zhang L: mir-30d Regulates multiple genes in the autophagy pathway and impairs autophagy process in human cancer cells. Biochem Biophys Res Commun 431: 617-622, 2013.
17. Walker JM: The bicinchoninic acid (BCA) assay for protein quantitation. Methods Mol Biol 32: 5-8, 1994.

18. Tusher VG, Tibshirani R and Chu G: Significance analysis of microarrays applied to the ionizing radiation response. Proc Natl Acad Sci USA 98: 5116-5121, 2001.

19. Livak KJ and Schmittgen TD: Analysis of relative gene expression data using real-time quantitative PCR and the 2(-Delta Delta C(T)) Method. Methods 25: 402-408, 2001

20. Xiao F, Zuo Z, Cai G, Kang S, Gao X and Li T: miRecords: An integrated resource for microRNA-target interactions. Nucleic Acids Res 37 (Database): D105-D110, 2009.

21. Hsu SD, Tseng YT, Shrestha S, Lin YL, Khaleel A, Chou CH, Chu CF, Huang HY, Lin CM, Ho SY, et al: miRTarBase update 2014: An information resource for experimentally validated miRNA-target interactions. Nucleic Acids Res 42 (D1): D78-D85, 2014.

22. Hashimoto D, Ohmuraya M, Hirota M, Yamamoto A, Suyama K, Ida S, Okumura Y, Takahashi E, Kido H, Araki K, et al: Involvement of autophagy in trypsinogen activation within the pancreatic acinar cells. J Cell Biol 181: 1065-1072, 2008.

23. Meijer AJ and Codogno P: Regulation and role of autophagy in mammalian cells. Int J Biochem Cell Biol 36: 2445-2462, 2004.

24. Mizushima N: Methods for monitoring autophagy. Int J Biochem Cell Biol 36: 2491-2502, 2004.

25. Xiao J, Zhu X, He B, Zhang Y, Kang B, Wang Z and Ni X MiR-204 regulates cardiomyocyte autophagy induced by ischemia-reperfusion through LC3-II. J Biomed Sci 18: 35, 2011.

26. Yamamoto A Cremona ML and Rothman JE: Autophagy-mediated clearance of huntingtin aggregates triggered by the insulin-signaling pathway. J Cell Biol 172: 719-731, 2006

27. Pan ST, Qin Y, Zhou ZW, He ZX, Zhang X, Yang T, Yang YX, Wang D, Qiu JX and Zhou SF: Plumbagin induces G2/M arrest, apoptosis, and autophagy via p38 MAPK- and $\mathrm{PI} 3 \mathrm{~K} / \mathrm{Akt} / \mathrm{mTOR}$-mediated pathways in human tongue squamous cell carcinoma cells. Drug Des Devel Ther 9: 1601-1626, 2015.

28. Heras-Sandoval D, Pérez-Rojas JM, Hernández-Damián J and Pedraza-Chaverri J: The role of PI3K/AKT/mTOR pathway in the modulation of autophagy and the clearance of protein aggregates in neurodegeneration. Cell Signal 26: 2694-2701, 2014.

29. Nakatogawa H: Two ubiquitin-like conjugation systems that mediate membrane formation during autophagy. Essays Biochem 55: 39-50, 2013.

30. Park S, Choi SG, Yoo SM, Son JH and Jung YK: Choline dehydrogenase interacts with SQSTM1/p62 to recruit LC3 and stimulate mitophagy. Autophagy 10: 1906-1920, 2014.

31. Schwartz-Roberts JL, Cook KL, Chen C, Shajahan-Haq AN, Axelrod M, Wärri A, Riggins RB, Jin L, Haddad BR, Kallakury BV, et al: Interferon regulatory factor-1 signaling regulates the switch between autophagy and apoptosis to determine breast cancer cell fate. Cancer Res 75: 1046-1055, 2015.

32. Joo JH, Dorsey FC, Joshi A, Hennessy-Walters KM, Rose KL, McCastlain K, Zhang J, Iyengar R, Jung CH, Suen DF, et al: Hsp90-Cdc37 chaperone complex regulates Ulk1- and Atg13-mediated mitophagy. Mol Cell 43: 572-585, 2011.

33. Merabova N, Sariyer IK, Saribas AS, Knezevic T, Gordon J, Turco MC, Rosati A, Weaver M, Landry J and Khalili K: WW domain of BAG3 is required for the induction of autophagy in glioma cells. J Cell Physiol 230: 831-841, 2015.

34. Neal MD, Sodhi CP, Dyer M, Craig BT, Good M, Jia H, Yazji I, Afrazi A, Richardson WM, Beer-Stolz D, et al: A critical role for TLR4 induction of autophagy in the regulation of enterocyte migration and the pathogenesis of necrotizing enterocolitis. J Immunol 190: 3541-3551, 2013

35. Giot L, Bader JS, Brouwer C, Chaudhuri A, Kuang B, Li Y, Hao YL, Ooi CE, Godwin B, Vitols E, et al: A protein interaction map of Drosophila melanogaster. Science 302: 1727-1736, 2003.

36. Li S, Armstrong CM, Bertin N, Ge H, Milstein S, Boxem M, Vidalain PO, Han JD, Chesneau A, Hao T, et al: A map of the interactome network of the metazoan $C$ elegans. Science 303: 540-543, 2004. 